Results With reference to our cases, we review the management protocols for ingested FB in paediatrics patients.

Conclusions Urgent Endoscopic removal (within 2 hours) is indicated in-

- All symptomatic patients - Sharp foreign body - Esophageal foreign body - Foreign body in the stomach if-

Age $<5$ years- $>2 \mathrm{~cm}$ in diameter and $>5 \mathrm{~cm}$ in length

Age $>5$ years- $>2.5 \mathrm{~cm}$ in diameter and $>6 \mathrm{~cm}$ in length

Non urgent endoscopic removal should be followed in-

- Magnetic foreign bodies within 24 hours - Ingested button batteries in stomach impacted for $>48$ hours - Blunt small nontoxic foreign body in the oesophagus for more than 24 hours.

\section{IDDF2018-ABS-0002 ESOPHAGEAL DUPLICATION CYST: DIAGNOSTIC DIFFICULTY IN PAEDIATRIC PATIENTS PRESENTING WITH RECURRENT CHEST INFECTION AND WHEEZE}

${ }^{1}$ Deepak Kumar*, ${ }^{2}$ Garima Gupta, ${ }^{1}$ Urmila Jhamb. 'Maulana Azad Medical College, New Delhi, India; ${ }^{2}$ NDMC and Hindu Rao Hospital, New Delhi, India

\subsection{6/gutjnl-2018-IDDFabstracts.58}

Background Esophageal Duplication Cyst (EDC) is a very rare congenital anomaly. It may present with respiratory and feeding difficulty depending upon the location in the oesophagus. We are reviewing this rare congenital malformation affecting the children who presented to us with the main symptom of recurrent chest infection and stridor.

Methods Our first patient is a 2 years 6 months old female child who had complaints of recurrent chest infections and stridor onset at 6 months of age. The child develops a cough, fever, fast breathing, retractions and stridor during each episode, these symptoms respond to the treatment for few weeks and then reoccurs. At 13 months of age, the child developed pneumonia, which was persisting, for which CECT chest was done. A cystic lesion adjacent to oesophagus was found suggestive of EDC. Our second case is an 11 months old female child who was well until 5 months of age had a history of recurrent chest infection and stridor on and off. The child develops a cough, fast breathing, retractions, noisy breathing (stridor) which response to medical treatment and then reappears after few weeks. On introduction of weaning food to the child, parents noted that child develops vomiting, regurgitates given a meal, and had choking like symptoms. These symptoms were more when given meal was semisolid (impacting) inconsistency and were increasing gradually. With these symptoms child brought to us. Chest X-ray done had shown tracheal deviation, CECT done revealed cystic lesion suggestive of EDC. In both cases, the diagnosis was confirmed by histopathological analysis of specimen removed surgically. On follow-up, patients were found healthy and asymptomatic.

Results Diagnosis of EDC is difficult but should be thought of after excluding common causes of persistent or recurrent wheeze, distress, stridor, vomiting and dysphagia. If missed, serious complications like rupture of the cyst, secondary infection, mediastinitis and malignant transformation can occur.

Conclusions EDC is a rare finding but should be included in patients presenting with recurrent wheeze and stridor, CECT is the diagnostic modality.

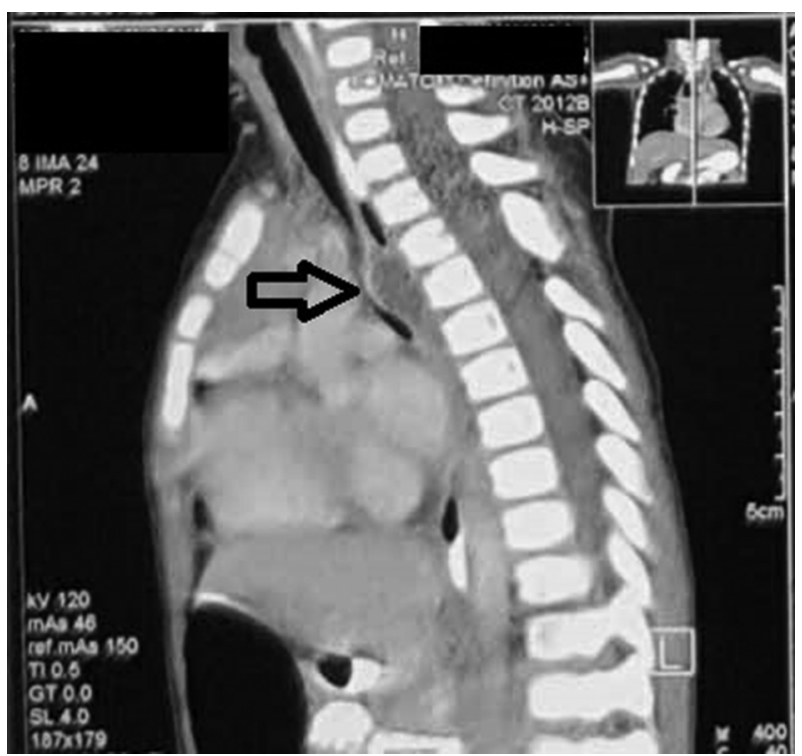

Abstract IDDF2018-ABS-0002 Figure 1

\section{IDDF2018-ABS-0003 COMPARISON OF PERFORMANCE OF IMMUNOASSAY BASED 'CELIAC CARD' AND HUMAN ANTI-TISSUE TRANSGLUTAMINASE ANTIBODY ELISA KIT FOR CELIAC DISEASE}

Manish Narang*, Chirag Verma, Dheeraj Shah. University College of Medical Sciences and GTB Hospital, Delhi, India

\subsection{6/gutjnl-2018-IDDFabstracts.59}

Background Serum levels of immunoglobulin A-tissue transglutaminase antibodies (IgA-tTG) by commercially available enzyme-linked immunosorbent technique (ELISA) are accepted as the best serology screening tool for celiac disease. ELISA based IgA-tTG tests are available only in centralised laboratories, and testing requires expert personnel in ideal conditions and therefore a long testing time. 'Celiac card' is recently developed rapid and sensitive one step immunoassay test based on detection of IgA. The objective of the study was to validate the diagnostic performance of immunoassay based 'celiac card' and compare it with human anti-tissue.

Methods This diagnostic accuracy study was carried out in Indian tertiary care hospital. Children aged between 1 to 12 years and considered to be high risk for celiac disease (recurrent diarrhoea/chronic diarrhoea/or recurrent abdominal pain along with poor weight gain or abdominal distension/or moderate to severe iron deficiency anaemia/or short stature) were recruited. Those with chronic gastrointestinal diseases like inflammatory bowel disease or already diagnosed celiac disease were excluded. Eligible participants were screened for IgA anti tTG antibodies by 'celiac card' and ELISA based 'Euroimmun kit'. Patients screened positive by either of the two diagnostic methods underwent upper gastrointestinal endoscopy and biopsy and diagnosis of celiac disease made as per Marsh grading.

Results Sensitivity and specificity of 'Celiac card' were calculated in 250 enrolled children and found out to be $92.31 \%$ and $98.28 \%$ respectively, which was similar to the ELISA based 'Euroimmun' kit. 